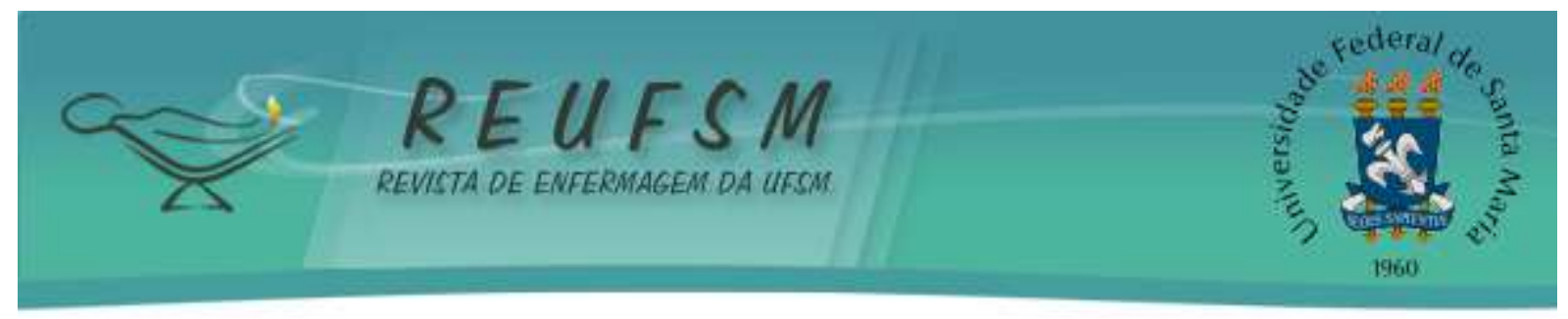

\title{
INCIDÊNCIA DA TUBERCULOSE E A CORRELAÇ̃̃O ENTRE DO TRATAMENTO E A CURA
}

\section{TUBERCULOSIS INCIDENCE AND THE CORRELATION BETWEEN TREATMENT AND HEALING}

\section{INCIDENCIA DE LA TUBERCULOSIS Y LA CORRELACIÓN ENTRE LA REALIZACIÓN DEL TRATAMIENTO Y LA CURA}

Doi: 10.5902/2179769230534

\author{
Jhenifer de Souza ${ }^{1}$ \\ Keurilene Sutil de Oliveira ${ }^{2}$ \\ Thaís Tania Ávila ${ }^{3}$ \\ Samia Regina de Quadros ${ }^{4}$ \\ Adriana Zilly ${ }^{5}$ \\ Reinaldo Antonio Silva-Sobrinho ${ }^{6}$
}

RESUMO: Objetivo: analisar a taxa de incidência da tuberculose, proporção de doentes e de casos curados nos municípios prioritários do estado do Paraná. Método: estudo ecológico baseado em dados secundários do período de 2001-2012, dos municípios prioritários para controle da tuberculose no estado do Paraná. Resultados: verificou-se um total de 15.053 casos novos de tuberculose no período analisado, 11.538 foram curados, sendo que quatro cidades não conseguiram atingir a meta de cura de $85 \%$ dos casos e 3.239 casos realizaram o Tratamento Diretamente Observado. Dois municípios apresentaram uma correlação forte (Curitiba r=0,7 e São José dos Pinhais com r=0,8). Conclusão: o estudo reforça a importância da atenção básica no controle da tuberculose, com a finalidade de contribuir para a diminuição da transmissão da doença, a partir da melhoria da adesão ao tratamento, reduzindo assim as taxas de abandono e o surgimento de cepas resistentes.

Descritores: Tuberculose; Incidência; Saúde Pública

ABSTRACT: Aim: to analyze the incidence of tuberculosis and the correlation between the cure of the new cases and the Directly Observed Treatment in priority municipalities in the state of Paraná. Methods: ecological study based on secondary data from the period 20012012, of the priority municipalities for tuberculosis control in the state of Paraná. Results: a total of 15,053 new cases of tuberculosis in the analyzed period were observed, 11,538 were cured, and four cities failed to reach the cure target of 85\% of the cases and only 3,239 cases underwent Directly Observed Treatment. Two municipalities presented a strong correlation

\footnotetext{
1 Enfermeira. Universidade Estadual do Oeste do Paraná. Foz do Iguaçu-PR, Brasil. E-mail: souza_jhenifer@yahoo.com.br

${ }_{2}^{2}$ Mestranda em Saúde Pública em Região de Fronteira. Especialista em Controle de Infecção Hospitalar. Universidade Estadual do Oeste do Paraná. Foz do Iguaçu-PR, Brasil. E-mail: keurisutil@gmail.com

${ }^{3}$ Mestranda em Ensino. Especialista em Urgência e Emergência. Universidade Estadual do oeste do Paraná. Foz do Iguaçu-PR, Brasil. E-mail: thaist_Avila@ hotmail.com

4 Enfermeira. Universidade Estadual do Oeste do Paraná. Foz do Iguaçu-PR, Brasil. E-mail: samiaquadros@hotmail.com

${ }^{5}$ Doutora em Ciências Biológicas pela Universidade Estadual de Maringá. Universidade Estadual do Oeste do Paraná. Foz do Iguaçu-PR, Brasil. E-mail: aazilly@ hotmail.com

${ }^{6}$ Pós-Doutor em Saúde Pública pela Universidade de São Paulo. Universidade Estadual do Oeste do Paraná. Foz do Iguaçu-PR, Brasil. E-mail: reisobrinho@unioeste.br
} 


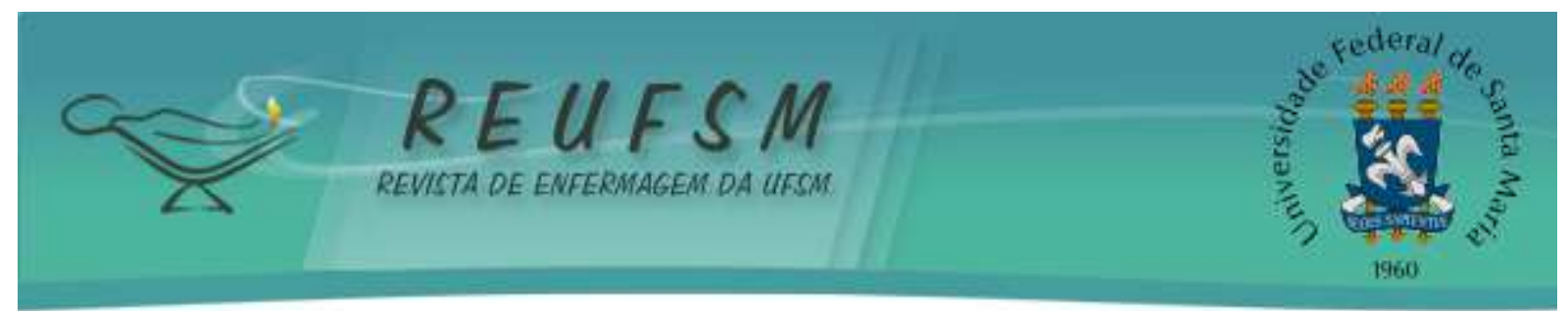

(Curitiba $r=0.7$ and São José dos Pinhais with $r=0.8$ ). Conclusion: the study reinforces the importance of basic care in TB control, with the aim of contributing to the reduction of disease transmission, by improving adherence to treatment, thus reducing abandonment rates and the emergence of resistant strains.

Descriptors: Tuberculosis; Incidence; Public health

RESUMEN: Objetivo: analizar la incidencia de la tuberculosis y la correlación entre la curación de los casos nuevos y la realización del Tratamiento Directamente Observado en ciudades prioritarias de la provincia de Paraná. Métodos: estudio ecológico basado en datos secundarios en el período de 2001-2012, de las ciudades prioritarias para el control de la tuberculosis en la provincia de Paraná. Resultados: se verificó un total de 15.053 casos nuevos de la tuberculosis en el período analizado, 11.538 fueron curados, pero cuatro ciudades no alcanzaron la meta de cura del 85\% de los casos y sólo 3.239 casos realizaron el Tratamiento Directo Observado. Las ciudades que presentaron una correlación fuerte: Curitiba $r=0,7$ y São José dos Pinhais con $r=0,8$. Conclusión: el estudio refuerza la importancia de la atención básica en el control de la tuberculosis, con el objetivo de contribuir con la disminución de la transmisión de la enfermedad, a partir de la ampliación de la adhesión al tratamiento, reduciendo así las tasas de abandono y el surgimiento de cepas más resistentes.

Descriptores: Tuberculosis; Incidencia; Salud pública

\section{INTRODUÇÃO}

A Organização Mundial da Saúde (OMS) considera a Tuberculose (TB) como um problema de saúde pública, sendo uma das principais causas de morte por doença infecciosa em adultos que se mantém nos países em desenvolvimento. ${ }^{1}$

Acrescenta-se que, nos últimos cinco anos, a doença foi responsável por mais óbitos que o Vírus da Imunodeficiência Humana (HIV), estando entre as 10 principais causas de morte no mundo. Registros preliminares do Ministério da Saúde apontam que 84.735 mil pessoas em 2017 foram registradas como casos novos no Brasil. ${ }^{2}$

No ano anterior, em 2016, foram diagnosticados e registrados 66.796 casos novos e 12.809 casos de retratamento de TB no Brasil, ${ }^{3}$ sendo que no mesmo ano o país se encontrava ocupando a $20^{\mathrm{a}}$ posição na classificação de carga da doença. ${ }^{4} \mathrm{~A}$ incidência de casos de TB no Brasil reduziu no período de 2007 a 2016, apresentando uma variação média anual de $-1,7 \%$, passando de 37,9/100 mil habitantes em 2007 para 32,4/100 mil habitantes em 2016. No Paraná, em 2016, a taxa de incidência da TB foi de 18,9/100 mil habitantes para todas as formas de TB diagnosticadas que foram registradas no Sistema de Informação de Agravos de Notificação (SINAN). ${ }^{3}$

No Brasil o tratamento da doença é disponibilizado na rede pública de saúde e a estratégia recomendada é o Tratamento Diretamente Observado (TDO), o que consiste na 


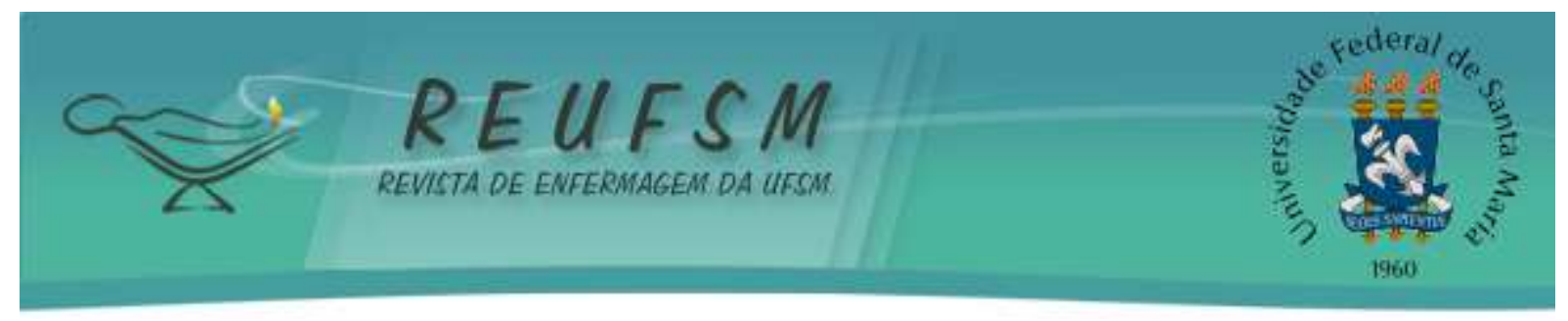

observação diária da ingestão dos medicamentos realizada por um membro da equipe de saúde. O TDO é uma ferramenta de gestão clínica em TB que tem potencial de promover o cuidado interdisciplinar, favorecendo a implementação de assistência voltada ao perfil dos doentes, especialmente aqueles em vulnerabilidade social e econômica. Desta maneira estabelece acesso, vínculo e acolhimento, sendo a participação dos doentes e familiares no tratamento, potencializador do processo de cura. ${ }^{5}$

Desde 1998, o Programa Nacional de Controle da Tuberculose (PNCT) intensificou e fortaleceu a realização de ações para a detecção e o tratamento dos casos ocorrendo, a partir de 2001, a realização da descentralização das ações de controle da TB para a Atenção Básica em Saúde com foco na redução da morbimortalidade e transmissão da doença. Para o alcance desta meta o enfoque central consistiu em estimular o implemento da cura dos doentes diagnosticados com TB. ${ }^{6}$

A despeito das estratégias adotadas para o enfrentamento da TB, o percentual de casos novos que realizam TDO é irregular nas várias regiões do país, variando de $27,6 \%$ na região norte a $42 \%$ na região Centro-Oeste. No estado do Paraná, o percentual de doentes atendidos com o TDO foi de $75,8 \%$, contudo ainda não alcançou o valor recomendado pelo indicador operacional que estabelece que, pelos menos $85 \%$ dos casos sejam curados entre o total de casos diagnosticados. ${ }^{7}$

Sobre as barreiras para a oferta do TDO, parece que variáveis ligadas ao envolvimento dos usuários, qualificação dos trabalhadores de saúde e disponibilidade de infraestrutura nas Unidades de Saúde estão entre aquelas que dificultam assegurar a realização da estratégia. ${ }^{5}$

Destaca-se que o Ministério da Saúde estabeleceu ações programáticas especiais, elegendo municípios prioritários como estratégia para controle epidemiológico da doença no país, recomendando o TDO como centro do plano terapêutico para todos os doentes de TB. Fez-se necessário um estudo para discutir o alcance destas ações no controle da TB no Estado do Paraná, por meio da análise dos indicadores epidemiológicos e operacionais.

Nesta perspectiva, o presente estudo teve por objetivo analisar a taxa de incidência da tuberculose, proporção de doentes e de casos curados nos municípios prioritários do estado do Paraná.

\section{MÉTODO}

Trata-se de um estudo ecológico baseado em dados secundários. Foram analisados os casos novos de TB em municípios que eram considerados prioritários para o controle da 


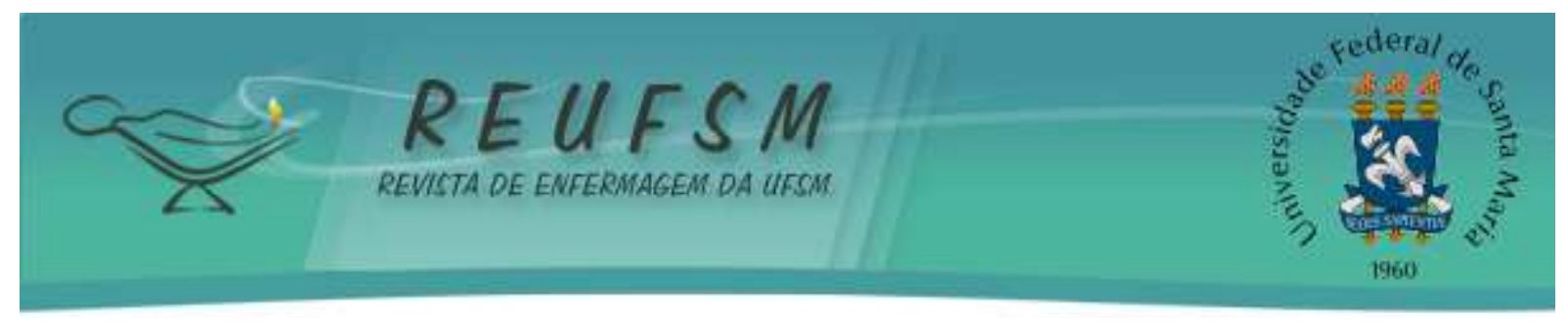

doença no estado do Paraná, sendo estes: Colombo, Curitiba, Foz do Iguaçu, Guarapuava, Londrina, Maringá, Paranaguá, Pinhais, Ponta Grossa e São Jose dos Pinhais.

O estado do Paraná está localizado na região sul do Brasil e conta com uma área de 199.307,922 km², possui 399 municípios com uma população de 10.444.526 habitantes, apresentando uma densidade demográfica de 52,40 habitantes $/ \mathrm{km}^{2} .8$

O estudo abrangeu um período de 12 anos ou seja de 2001 até 2012. As informações de morbidade foram buscadas no Sistema de Informação de Agravos de Notificação (SINAN) da Secretaria Estadual de Saúde do Paraná (SESA). Os dados demográficos referentes aos municípios foram obtidos por meio do Sistema de Informação público - TabNet Win32 versão 3.0, alimentado pelo Departamento de Informática do Sistema Único de Saúde (DATASUS) e Instituto Brasileiro de Geografia e Estatística (IBGE), de acordo com a população residente estimada para cada ano da série temporal. Por informação da SESA-PR, os dados de 2001 a 2012 haviam passado por uma ampla e profunda revisão, realizada pela própria SESA e repassada ao MS/DATASUS (e já estavam atualizadas no TabNet Win32 versão 3.0, assim os pesquisadores, optaram por esse período - visto a sabida qualidade dos dados).

A busca de dados nos sistemas de informação ocorreu no ano de 2017. No SINAN foi obtido o número bruto de casos notificados, curados e de doentes que realizaram o TDO.

Para este estudo foi construído um banco de dados por meio de planilhas, utilizando o programa Excel® (Microsoft Office 2010, Microsoft Corporation, EUA) que permitiu calcular três indicadores; 1) taxa de incidência de TB, 2) proporção de casos curados e 3) proporção de doentes de TB que realizaram o TDO, conforme Quadro 1.

No que se refere ao número de pacientes que realizaram o TDO, for registrado um total de 3.239 de 2001 a 2009, excluindo os anos de 2010 a 2012, pois ocorreram algumas discrepâncias entre os resultados obtidos, evidenciando prováveis erros de anotação/digitação destes casos de TB que realizaram o TDO em relação à população de cada ano nos municípios. 


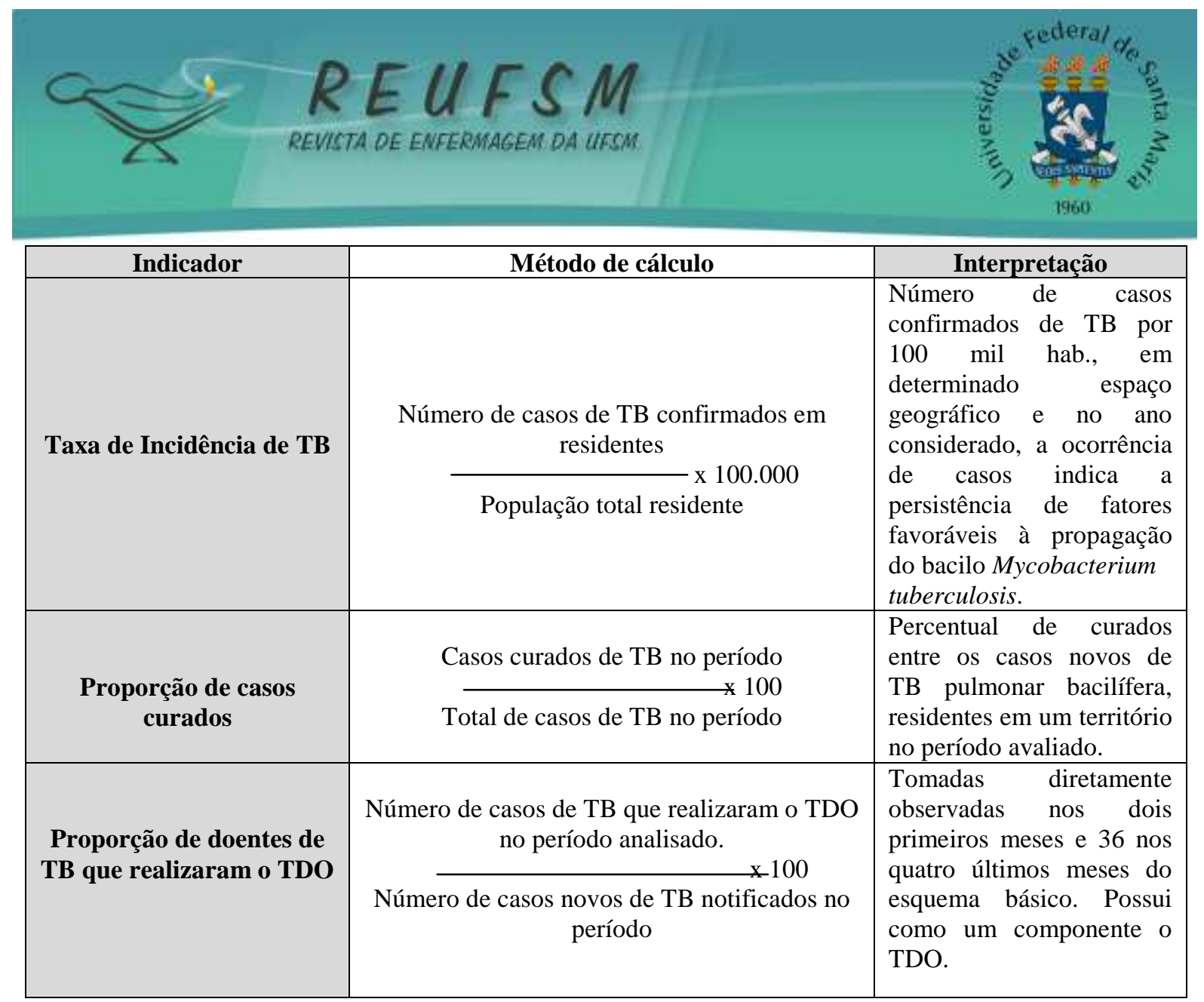

Quadro 1 - Indicadores para cálculo e análise da proporção de casos e incidência de TB nos municípios prioritários do estado do Paraná no período de 2001 a 2012

Fonte: Ministério da Saúde, 2014. ${ }^{9}$

Nota: Os anos de 2010 a 2012 foram excluídos, porque o registro de casos que realizaram TDO era maior do que os casos notificados.

Para analisar a correlação entre a realização do TDO e a cura, foi calculada a Correlação Linear de Pearson, que é uma medida do grau de relação linear entre duas variáveis quantitativas, utilizando o software Statatisca versão12/StatSoft.

O estudo foi aprovado por Comitê de Ética em Pesquisa e seguiu os preceitos éticos da Resolução 466/12, do Conselho Nacional da Saúde. Sob o parecer no 102/2013, processo CAAE 18018013.5.0000.0107, emitido no dia 25 de julho de 2013.

\section{RESULTADOS}

No estudo foi identificado um total de 15.053 casos novos de TB registrados no período de 2001 a 2012, nos municípios prioritários do estado do Paraná.

Conforme demonstrado na Tabela1, a seguir Paranaguá se destacou com o mais elevado coeficiente de incidência do período entre os municípios em estudo, sendo que em 2008 o coeficiente chegou a 107 casos por 100 mil habitantes. Por outro lado, a observou-se que a cidade de Maringá apresentou menores coeficientes de incidência. 


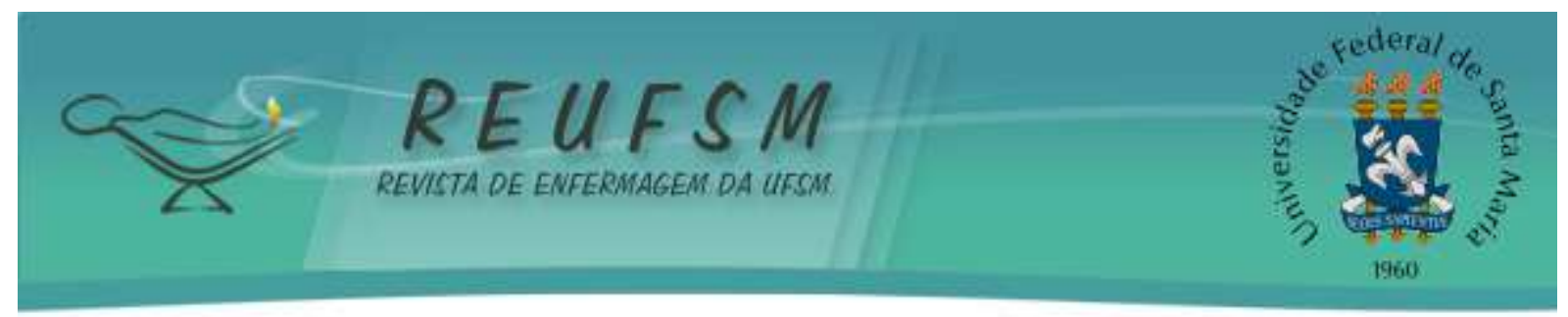

Tabela 1 - Incidência de casos de tuberculose nos municípios prioritários do estado do

Paraná no período de 2001 a 2012. Paraná, Brasil, 2017

\begin{tabular}{|c|c|c|c|c|c|c|c|c|c|c|c|c|}
\hline & 2001 & 2002 & 2003 & 2004 & 2005 & 2006 & 2007 & 2008 & 2009 & 2010 & 2011 & 2012 \\
\hline \multicolumn{13}{|c|}{ Incidência de casos/100.000 habitantes $(N=15.053)$} \\
\hline Colombo & 26 & 35 & 36 & 27 & 24 & 30 & 24 & 22 & 15 & 22 & 32 & 26 \\
\hline Curitiba & 30 & 33 & 32 & 29 & 31 & 25 & 25 & 24 & 24 & 25 & 23 & 22 \\
\hline Foz do Iguaçu & 84 & 53 & 62 & 49 & 47 & 44 & 42 & 47 & 32 & 42 & 47 & 52 \\
\hline Guarapuava & 33 & 26 & 35 & 31 & 20 & 28 & 19 & 21 & 17 & 10 & 11 & 12 \\
\hline Londrina & 32 & 23 & 29 & 25 & 26 & 27 & 21 & 22 & 25 & 23 & 26 & 24 \\
\hline Maringá & 25 & 25 & 18 & 21 & 14 & 16 & 20 & 22 & 27 & 21 & 22 & 13 \\
\hline Paranaguá & 78 & 102 & 89 & 86 & 101 & 76 & 93 & 107 & 98 & 100 & 88 & 86 \\
\hline Pinhais & 75 & 62 & 88 & 78 & 66 & 59 & 62 & 56 & 81 & 62 & 64 & 64 \\
\hline $\begin{array}{l}\text { São José dos } \\
\text { Pinhais }\end{array}$ & 25 & 23 & 30 & 29 & 26 & 22 & 31 & 27 & 35 & 26 & 28 & 23 \\
\hline Ponta Grossa & 18 & 30 & 33 & 20 & 26 & 24 & 32 & 31 & 24 & 20 & 21 & 23 \\
\hline
\end{tabular}

Em relação aos casos curados no estado do Paraná entre 2001 a 2012, foram

identificados no estudo 11.538 pacientes. Quando calculado a proporção de cura, cinco municípios atingiram $85 \%$ de casos curados entre os notificados, índice mínimo preconizado pelo Ministério da Saúde, ${ }^{5}$ sendo que Paranaguá foi o único município que conseguiu curar todos os doentes que notificou durante o período. Curitiba, Foz do Iguaçu, Londrina e Pinhais não atingiram a proporção mínima em nenhum ano do estudo (Tabela 2).

Tabela 2 - Proporção de casos notificados de tuberculose curados nos municípios prioritários do estado do Paraná no período de 2001 a 2012. Paraná, Brasil, 2017

\begin{tabular}{|c|c|c|c|c|c|c|c|c|c|c|c|c|}
\hline & 2001 & 2002 & 2003 & 2004 & 2005 & 2006 & 2007 & 2008 & 2009 & 2010 & 2011 & 2012 \\
\hline \multicolumn{13}{|c|}{ Percentual (\%) } \\
\hline Colombo & 82 & 90 & 78 & 68 & 67 & 74 & 74 & 78 & 74 & 64 & 79 & 70 \\
\hline Curitiba & 74 & 71 & 69 & 70 & 74 & 70 & 67 & 69 & 70 & 76 & 77 & 76 \\
\hline $\begin{array}{ll}\text { Foz } & \text { do } \\
\text { Iguaçu } & \end{array}$ & 67 & 64 & 64 & 70 & 72 & 81 & 72 & 72 & 77 & 70 & 72 & 68 \\
\hline Guarapuava & 77 & 93 & 77 & 78 & 91 & 89 & 78 & 88 & 83 & 100 & 95 & 95 \\
\hline Londrina & 78 & 76 & 77 & 81 & 69 & 71 & 72 & 75 & 68 & 66 & 80 & 74 \\
\hline Maringá & 84 & 87 & 71 & 71 & 70 & 74 & 64 & 80 & 81 & 84 & 70 & 67 \\
\hline Paranaguá & 100 & 100 & 100 & 100 & 100 & 100 & 100 & 100 & 100 & 100 & 100 & 100 \\
\hline Pinhais & 70 & 75 & 63 & 61 & 80 & 71 & 80 & 74 & 77 & 75 & 78 & 77 \\
\hline $\begin{array}{l}\text { São José dos } \\
\text { Pinhais }\end{array}$ & 87 & 73 & 81 & 77 & 71 & 74 & 77 & 70 & 85 & 84 & 74 & 76 \\
\hline $\begin{array}{l}\text { Ponta } \\
\text { Grossa }\end{array}$ & 80 & 86 & 83 & 75 & 81 & 70 & 90 & 82 & 83 & 78 & 86 & 82 \\
\hline
\end{tabular}

Fonte: Ministério da Saúde, 2017. 


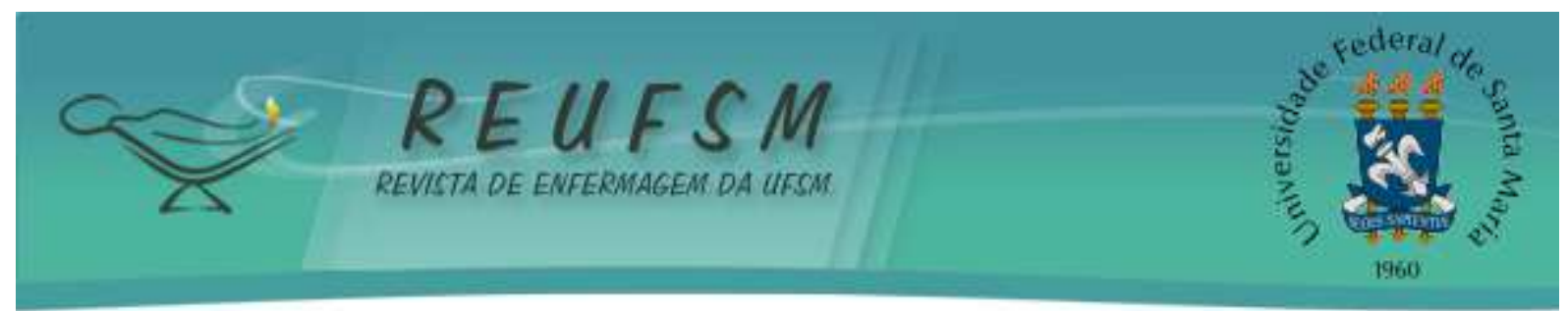

Conforme Tabela 3, Foz do Iguaçu e Ponta Grossa por quatro anos não apresentaram nenhum doente com TB que tivesse realizado o TDO. Quando considerado o indicador ano a ano, o município de Pinhas foi o que obteve maior proporção de tratamento com o TDO e Maringá alcançou a mais alta proporção de casos nesta modalidade de acompanhamento.

Tabela 3 - Proporção de doentes de tuberculose que realizaram o tratamento diretamente observado nos municípios prioritários do estado do Paraná no período de 2001 a 2009. Paraná, Brasil, 2017

\begin{tabular}{|c|c|c|c|c|c|c|c|c|c|}
\hline & 2001 & 2002 & 2003 & 2004 & 2005 & 2006 & 2007 & 2008 & 2009 \\
\hline \multicolumn{10}{|c|}{ Percentual (\%) } \\
\hline Colombo & 2 & 4 & 5 & 1 & 12 & 33 & 44 & 37 & 44 \\
\hline Curitiba & 11 & 13 & 19 & 16 & 13 & 23 & 66 & 62 & 57 \\
\hline Foz do Iguaçu & 0 & 0 & 1 & 0 & 0 & 2 & 49 & 51 & 65 \\
\hline Guarapuava & 25 & 44 & 32 & 64 & 88 & 72 & 75 & 44 & 50 \\
\hline Londrina & 1 & 0 & 2 & 2 & 5 & 10 & 24 & 44 & 28 \\
\hline Maringá & 4 & 1 & 1 & 0 & 4 & 20 & 72 & 76 & 83 \\
\hline Paranaguá & 0 & 1 & 0 & 2 & 3 & 5 & 35 & 40 & 77 \\
\hline Pinhais & 50 & 60 & 51 & 40 & 54 & 72 & 82 & 81 & 73 \\
\hline $\begin{array}{l}\text { São José dos } \\
\text { Pinhais }\end{array}$ & 24 & 15 & 16 & 21 & 35 & 42 & 80 & 68 & 74 \\
\hline Ponta Grossa & 0 & 0 & 0 & 0 & 2 & 9 & 26 & 21 & 33 \\
\hline
\end{tabular}

Fonte: Ministério da Saúde, 2017.

Nota: Os anos de 2010 a 2012 foram excluídos, porque o registro de casos que realizaram TDO era maior do que os casos notificados.

Os dados da Figura 1 representam os valores obtidos para os Coeficientes de Correlação Linear de Pearson entre a variável realização do TDO e cura, Foz do Iguaçu, Maringá e Londrina apresentaram correlação fraca ( $r$ entre 3 e 5). Nesta análise, foi possível detectar dois municípios do estado com uma correlação forte entre a realização do TDO e a cura - Curitiba e São José dos Pinhais com r entre 7 e 9. 

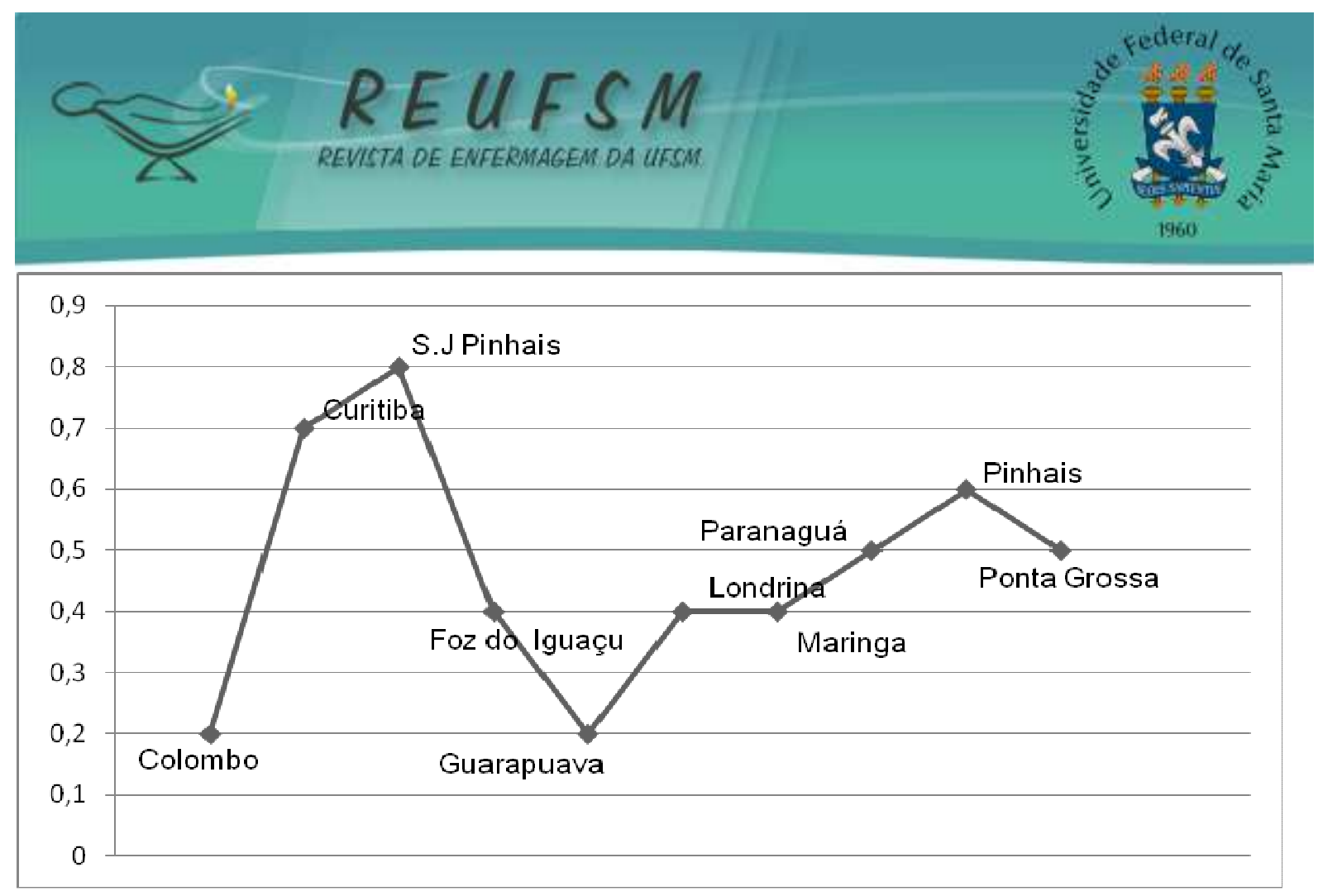

Nota: 0.9 para mais ou para menos indica uma correlação muito forte, 0.7 a 0.9 positivo ou negativo indica uma correlação forte, 0.5 a 0.7 positivo ou negativo indica uma correlação moderada, 0.3 a 0.5 positivo ou negativo indica uma correlação fraca, 0 a 0.3 positivo ou negativo indica uma correlação desprezível

Figura 1 - Coeficientes de correlação linear de Pearson entre a variável de cura e realização do tratamento diretamente observado nos casos de Tuberculose nos municípios prioritários do estado do Paraná no período de 2001 a 2012. Paraná, Brasil, 2017

\section{DISCUSSÃO}

No presente estudo, o coeficiente incidência em municípios prioritários do Paraná, foi superior ao coeficiente do Brasil em 2012, 36,8 casos para 100 mil habitantes. O alto no número de casos na grande maioria dos anos, nos municípios deste estudo, mostra que a redução da incidência pode demorar mais do que o previsto (ano 2035), visto que a taxa de incidência mundial vem diminuindo lentamente, menos de $1 \%$ ao ano.,

O município de Paranaguá localiza-se em região portuária, apresentando concomitante, elevado número de casos de TB e da Síndrome da imunodeficiência adquirida (Aids), além de outros determinantes, como a baixa escolaridade e renda, aspectos que potencializam surgimento de casos novos de TB e que podem justificar o registro do maior coeficiente de incidência do Paraná. Porém, o serviço de atenção às pessoas adoecidas por TB neste município, foi capaz de promover o tratamento e alta por cura para100\% dos doentes. Segundo estudo realizado em Paranaguá, a introdução da estratégia da busca ativa, em paralelo com a adoção das demais ações preconizadas pela estratégia do TDO, foi um dos fatores responsáveis pelo aumento da detecção e a cura de casos no município. ${ }^{10}$ 


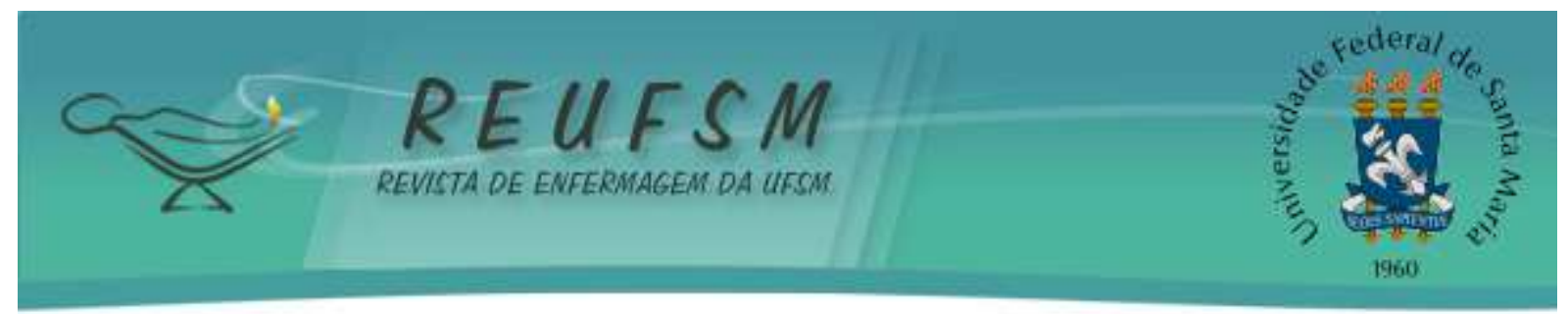

Na cidade de Foz do Iguaçu até o ano de 2010, o atendimento aos doentes contava com o Programa de TB centralizado, sendo que somente no ano de 2011 foi estabelecida a descentralização do atendimento para as Unidades de Saúde de Atenção Básica. E importante ressaltar que este município faz fronteira com Paraguai e Argentina, e a heterogeneidade populacional e o fluxo de pessoas entre os países são fatores que também impedem a detecção precoce da doença e o tratamento correto, atribuindo maior vulnerabilidade aos indivíduos da fronteira. ${ }^{11}$

Portanto, o alto coeficiente de incidência em alguns municípios pode se justificar pelas falhas na detecção precoce dos doentes e no funcionamento dos programas nacional e estadual de controle da TB. Infelizmente, mesmo sendo conhecidas as formas de contágio e de tratamento pelos profissionais de saúde e a alta possibilidade de cura em seis meses, a enfermidade ainda gera preconceito e estigma entre os doentes e a população em geral, o que provoca o afastamento dos pacientes, podendo influenciar diretamente na adesão ao tratamento. ${ }^{12}$

Além disso, o MS desde 2001 programou alguns aspectos a serem cumpridos em relação à detecção precoce dos sintomáticos respiratórios. Dentre eles: a descentralização, melhoria da vigilância epidemiológica e do sistema de informação, ampliação e qualificação da rede de laboratórios, garantia de acesso ao tratamento e capacitação de recursos humanos e incorporação de ações de controle da doença na Estratégia Saúde da Família. ${ }^{13}$

Ao que se refere aos casos curados de TB, a meta de cura é de $85 \%$ estabelecido pela OMS, sendo o indicador "proporção de casos curados entre casos novos diagnosticados" de grande utilidade para subsidiar a avaliação da efetividade do tratamento. Este indicador apontou falhas graves nos Programas Municipais de Controle da Tuberculose de quatro municípios do estado, os quais não atingiram a meta de cura em nenhum ano do estudo, sugerindo inconsistência na realização do TDO como, por exemplo, falta de acolhimento e abandono dos doentes antes de completar os seis meses de tratamento. ${ }^{5,9}$

Acrescenta-se que apenas o município de Paranaguá atingiu, durante todos os anos $100 \%$ de casos curados, superando a meta preconizada. Os municípios de Curitiba, Foz do Iguaçu, Londrina e Pinhais não conseguiram curar, pelo menos, $85 \%$ dos casos em nenhum ano do estudo. Em 2010 no estado do Paraná, a proporção de cura entre os casos notificados alcançou somente $76,4 \%$ de cura, ${ }^{14}$ indicando baixa capacidade em promover um desfecho para os casos de TB. 


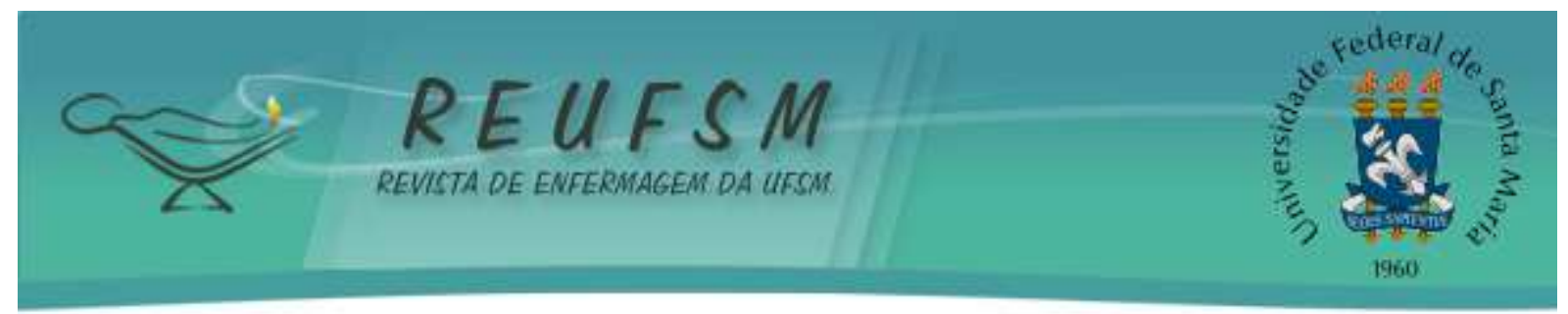

Quanto à proporção de curados que realizaram o TDO foi notoriamente baixo em todos os municípios prioritários, apesar de ser a estratégia estabelecida pela OMS e considerada essencial para promover a cura da TB. Para implantar efetivamente o TDO, é fundamental a reorganização da assistência prestada aos doentes e para tanto, sugere-se o fortalecimento dos programas municipais, além de capacitação dos profissionais de saúde para detecção precoce de sintomáticos, implementação na cobertura da Atenção Básica em Saúde, garantia de diagnóstico e tratamento precoce perto de sua residência, conforme as necessidades e condições do doente ${ }^{3}$ envolvendo os Agentes Comunitários de Saúde que são facilitadores da promoção e vigilância da saúde. ${ }^{15}$

Uma importante descoberta foi que existe correlação entre o TDO e o desfecho cura, e esta evidência indica que os municípios devem investir na estrutura de recursos humanos, físicos, organizacionais e no processo da atenção em TB, para garantir o acesso ao TDO que é potencialmente efetivo para a cura do doente. ${ }^{16-18}$

Por fim, salienta-se que houve baixa proporção de casos acompanhados por meio do TDO se comparado com o número de casos notificados, possivelmente por falha operacional, pela subnotificação ou por informações não registradas no SINAN. Portanto, faz-se necessário a melhoria no controle da TB e da qualidade de registro dos dados.

Vale salientar algumas limitações desta pesquisa, entre elas as relacionadas a estudos realizados com dados secundários, como possível incongruências no acompanhamento dos doentes, subnotificação e viés ecológico. Contudo, recursos metodológicos foram utilizados para promover a qualidade das evidências como, por exemplo, o uso de dados revisados pela SESA-PR.

\section{CONCLUSÃO}

Os municípios prioritários para o controle da TB no estado do Paraná mantêm elevados coeficientes de incidência da doença e, como fator complicador o percentual de cura não tem alçado os $85 \%$ propostos pelas organizações internacionais na maioria dos anos estudados - 2001 a 2012. De tal modo, torna-se fundamental a reorganização das políticas públicas municipais direcionadas ao controle da TB e da promoção da saúde.

Constata-se a necessidade de estratégias adicionais de promoção e vigilância em saúde, adotar medidas intersetoriais de promoção e vigilância, atuando de forma articulada a fim de cobrir 


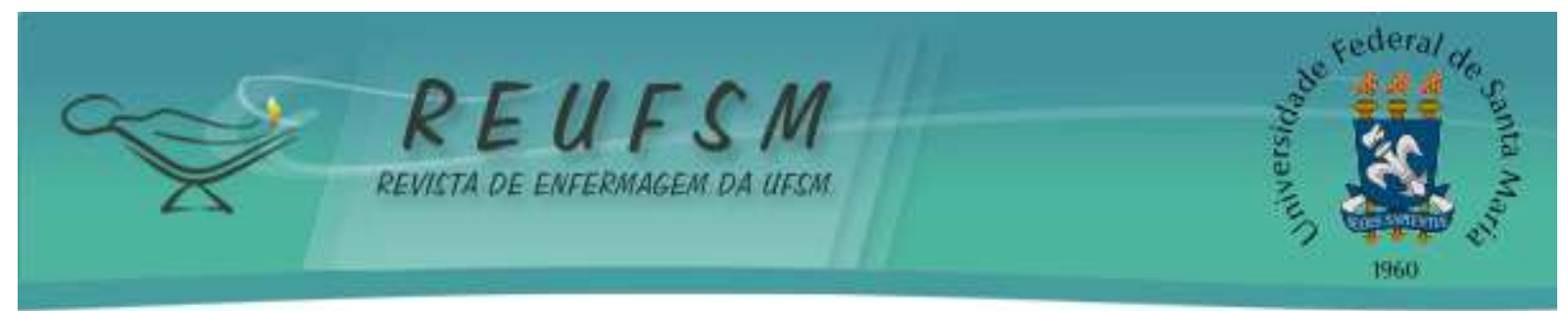

os vários aspectos que envolvem o controle de $\mathrm{TB}$, para alcançar a diminuição de incidência e aumento da cura, conforme os valores estabelecidos pelo MS para o controle da doença.

Também é importante a realização de novas pesquisas que avaliem o tratamento prestado ao doente, possíveis razões de abandono antes de atingir a cura, e a forma que esta sendo a realização da busca ativa de sintomáticos respiratórios nos municípios, as quais podem ser úteis no sentido de melhorar o monitoramento da doença.

O presente estudo reforça a importância da atenção básica para o controle da TB com a finalidade de contribuir para a diminuição da transmissão da doença, a partir da melhoria da adesão ao tratamento, reduzindo assim as taxas de abandono e o surgimento de cepas resistentes.

\section{REFERÊNCIAS}

1. Heck MA, Costa JSD, Nunes MF. Avaliação do programa de tuberculose em Sapucaia do Sul (RS): indicadores, 2000-2008. Ciênc Saúde Colet [Internet]. 2013 [acesso em 2017 jul 11];18(2):481-8. Disponível em: http://www.scielo.br/scielo.php?script=sci_arttext\&pid=S1413-81232013000200019.

2. Brasil. Ministério da Saúde/SVS - Sistema de Informação de Agravos de Notificação Sinan Net. Casos confirmados por ano diagnóstico segundo tipo de entrada [Internet]. [acesso em 2018 jun 12]. Disponível em http://tabnet.datasus.gov.br/cgi/tabcgi.exe?sinannet/cnv/tubercbr.def.

3. Brasil. Ministério da Saúde. Secretaria de Vigilância em Saúde. Indicadores prioritários para o monitoramento do Plano Nacional pelo fim da tuberculose como problema de saúde Pública no Brasil. Bolepidemiol. 2017;48(8):1-11.

4. Brasil. Ministério da Saúde. Secretaria de Vigilância em Saúde. Plano Nacional pelo fim da tuberculose. $1^{\text {a}}$ ed.Brasília: Ministério da Saúde; 2017.

5. Shuhama BV, Silva LMC, Andrade RLP, Palha PF, Hino P, Souza KMJ. Evaluation of the directly observed therapy for treating tuberculosis according to the dimensions of policy transfer. Rev Esc Enferm USP. 2017;51e03275.

6. Brasil. Secretaria de Vigilância em Saúde. Departamento de Vigilância Epidemiológica. Brasil livre da Tuberculose. Plano Nacional pelo fim da tuberculose como problema de saúde pública [Internet]. Brasília: Ministério da Saúde; 2017 [acesso em 2018 jan 22]. Disponível em: http://portalarquivos.saude.gov.br/images/pdf/2017/fevereiro/24/Plano-NacionalTuberculose.pdf.

7. Brasil. Ministério da Saúde. Implantação do Plano Nacional pelo Fim da Tuberculose como Problema de Saúde Pública no Brasil: primeiros passos rumo ao alcance das metas. Boletim Epidemiológico [Internet]. 2018;49(11):1-18. Disponível em: http://portalarquivos2.saude.gov.br/images/pdf/2018/marco/26/2018-009.pdf.

8. Instituto Brasileiro de Geografia e Estatística (IBGE). Censo Demográfico 2010 [Internet]. [acesso em 2017 jul 12]. Disponível em: http://cidades.ibge.gov.br. 


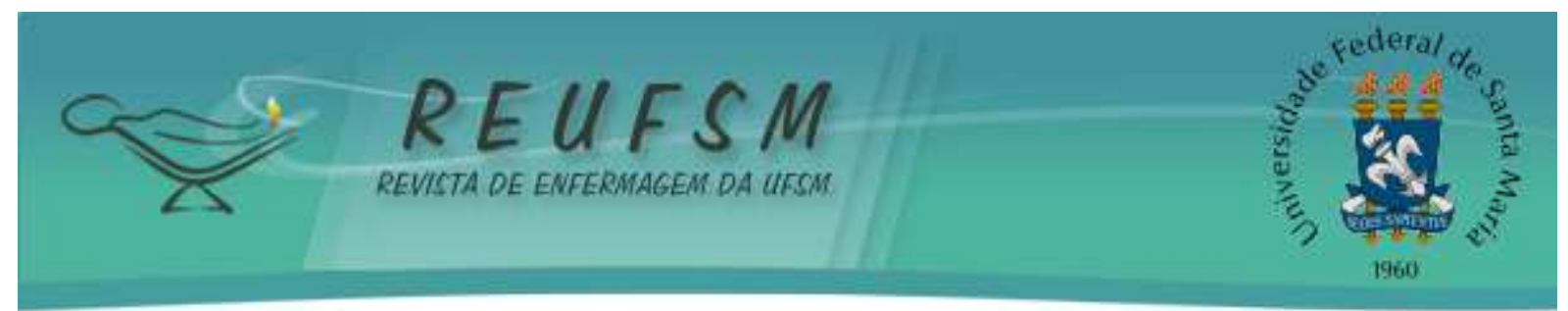

9. Brasil. Secretaria de Vigilância em Saúde. Departamento de Vigilância das Doenças Transmissíveis. Panorama da tuberculose no Brasil: indicadores epidemiológicos e operacionais [Internet]. Brasília: Ministério da Saúde; 2014 [acesso em 2017 jul 12]. Disponível em: http://bvsms.saude.gov.br/bvs/publicacoes/panorama\%20tuberculose\%20brasil_2014.pdf.

10. Gabardo BMA. Sintomáticos respiratórios em município de elevada incidência de Tuberculose [tese]. Curitiba: Universidade Federal do Paraná, Programa de pós-graduação em Medicina Interna; 2014. 107 p.

11. Giovanella L, Guimarães L, Nogueira VMR, Lobato LVC, Damacena GN. Saúde nas fronteiras: acesso e demandas de estrangeiros e brasileiros não residentes ao SUS nas cidades de fronteira com países do MERCOSUL na perspectiva dos secretários municipais de saúde. Cad Saúde Pública.2007;23 Sup 2:251-66.

12. Rodrigues ALI, Motta SCM, Ferreira AM. Representações sociais da tuberculose por enfermeiros. Rev Bras Enferm [Internet]. 2016 [acesso em 2017 jul 14];69(3):532-7. Disponível em: http://www.scielo.br/pdf/reben/v69n3/0034-7167-reben-69-03-0532.pdf.

13. Furlan MCR, Gonzales RIC, Marcon SS. Desempenho dos serviços de controle da tuberculose em municípios do Paraná: enfoque na família. Rev Gaúcha Enferm. 2015;36(N Esp):102-10.

14. Paraná. Secretaria Estadual da Saúde. Secretaria de Vigilância à Saúde. SINAN TB. Curitiba: Programa Estadual de Controle da Tuberculose/ DVVTR/CEPI; 2014.

15. Maciel ELN, Vieira RCA, Milani EC, Brasil M, Fregona G, Dietze R. O agente comunitário de saúde no controle da tuberculose: conhecimentos e percepções. Cad Saúde Pública [Internet]. 2008 [acesso em 2017 jul 15];24(6):1377-86. Disponível em: http://www.scielo.br/scielo.php?script=sci_arttext\&pid=S0102-311X2008000600018.

16. Silva Sobrinho RA, Wysocki AD, Scatena LM, Galvão ES, Beraldo AA, Andrade RLS, et al. Assessment of primary health care in the treatment of tuberculosis in a brazilian locality of the international triple frontier. Open Journal of Nursing. 2017;11:124-34.

17. Sales CF, Castro ME, Bezerra LG, Viana JG, Nunes FAM. Ações de controle da tuberculose: análise a partir do programa de melhoria do acesso e da qualidade da atenção básica. Texto Contexto \& Enferm [Internet]. 2016 [acesso em 2018 jul 09];25(4):e4660015. Disponivel em: http://www.scielo.br/scielo.php?script=sci_arttext\&pid=S010407072016000400323\&lng=en.

18. Couto DS, Carvalho RN, Azevedo EB, Moraes MN, Pinheiro PGOD, Faustino EB. Fatores determinantes para o abandono do tratamento da tuberculose: representações dos usuários de um hospital público. Saúde Debate. 2014; 38(102):572-81.

Data de submissão: 22/12/2017

Data de aceite: $17 / 07 / 2018$

Autor correspondente: Jhenifer de Souza

E-mail: Souza_jhenifer@yahoo.com.br

Endereço: rua victorio basso, 1140

CEP: $85859-72$ 